Received: 05.11 .2018

Revised: 19.11 .2018

Accepted: 30.11 .2018

DOI: $10.17804 / 2410-9908.2018 .6 .117-125$

\title{
THIN FILM FeGa-FeCoGa/Metglas/LGT STRUCTURES FOR MAGNETOELECTRIC MAGNETIC FIELD SENSORS
}

\author{
A. P. $\operatorname{Nosov}^{1,2, a)^{*}}$, I. V. Gribov ${ }^{1, b)}$, N. A. Moskvina ${ }^{1, c)}$, \\ A. V. Druzhinin ${ }^{1, \text { d) }}$, V. I. Osotov ${ }^{1, \text { e) }}$ \\ ${ }^{1}$ M.N. Miheev Institute of Metal Physics of Ural Branch of Russian Academy of Sciences, \\ 18 S. Kovalevskoi st., 620108, Ekaterinburg, Russian Federation, \\ ${ }^{2}$ Ural Federal University 19 Mira st., 620002, Ekaterinburg, Russian Federation \\ a) (iD https://orcid.org/0000-0003-1475-0059 @ nossov@imp.uran.ru; \\ b) (ID https://orcid.org/0000-0002-5067-2281 gri@imp.uran.ru; \\ c) (D) https://orcid.org/0000-0003-4285-0399 @ nat@imp.uran.ru; \\ d) (ID https://orcid.org/0000-0001-9944-096X @ druzhinin@imp.uran.ru; \\ e)国osotov@imp.uran.ru \\ *Corresponding author. E-mail: nossov@imp.uran.ru \\ Address for correspondence: S. Kovalevskoi str. 18, 620108, Ekaterinburg, Russian Federation \\ Tel.: +7 (343) 37479 22; fax: +7 (343) 3745244
}

The paper studies the influence of the composition of a graded magnetostrictive layer on the magnetoelectric effect in three-layered laminated structures of the type composite magnetostrictive ferromagnet / ferroelectric / composite magnetostrictive ferromagnet. A single crystal of $\mathrm{La}_{3} \mathrm{Ga}_{5.5} \mathrm{Ta}_{0.5} \mathrm{O}_{14}$ (lanthanum gallium tantalite) is used as the ferroelectric. The graded magnetostrictive layer consists of a Metglas-type amorphous ribbon with magnetostrictive Galfenol thin films of either $\mathrm{Fe}_{0.72} \mathrm{Ga}_{0.28}$ or $\mathrm{Fe}_{0.62} \mathrm{Co}_{0.19} \mathrm{Ga}_{0.19}$ compositions deposited on it by pulsed laser deposition. The $d c$ and $a c$ magnetic field dependences of the magnetoelectric effect are investigated in the frequency range from $20 \mathrm{~Hz}$ to $10 \mathrm{kHz}$. Magnetic noise is investigated at frequencies ranging between 0.5 and $14 \mathrm{~Hz}$. It is shown experimentally that the deposition of the $\mathrm{Fe}_{0.62} \mathrm{Co}_{0.19} \mathrm{Ga}_{0.19}$ films increases the value of the magnetoelectric voltage coefficient and decreases magnetic noise. The results can be useful in the development of magnetoelectric sensors of $d c$ and $a c$ magnetic fields for nondestructive testing at elevated temperatures.

Keywords: nondestructive testing, magnetic field sensor, magnetoelectric effect, amorphous alloy, thin films, Galfenol, lanthanum gallium tantalate.

\section{Acknowledgment}

The work was performed within the state assignment from the Ministry of Education of Russia (Spin, No. AAAA-A18-118020290104-2).

\section{References}

1. Lawes G., Srinivasan G. Introduction to magnetoelectric coupling and multiferroic films. J. Phys. D: Appl. Phys., 2011, vol. 44. pp. 243001. DOI: 10.1088/0022-3727/44/24/243001.

2. Petrov V.M., Srinivasan G. Enhancement of magnetoelectric coupling in functionally graded ferroelectric and ferromagnetic bilayers. Phys. Rev. B, 2008, vol.78, pp. 84421. DOI: 10.1103/PhysRevB.78.184421. 
3. Mandal S.K., Sreenivasulu G., Petrov V.M., Srinivasan G. Flexural deformation in a compositionally stepped ferrite and magnetoelectric effects in a composite with piezoelectrics. Appl. Phys. Lett., 2010, vol. 96, pp. 192502. DOI: 10.1063/1.3428774.

4. Magnetic Alloy 2605SA1 (iron-based). Tech. Bulletin, ref: 2605SA106192009, Metglas Inc., Conway, SC, 2009.

5. $\quad$ Passamani E.C., Larica C., Moscon P.S., Zelis P.M., Sanchez F.H. Out-of-plane anisotropy and low field induced magnetic domain reorientation in Al/Metglas-2605S2/Al trilayer sensors. J. Appl. Phys., 2011, vol. 110, pp. 043906. DOI: 10.1063/1.3622339.

6. Gribov I.V., Osotov V.I., Nosov A.P., Petrov V.M., Sreenivasulu G., Srinivasan G. Magneto-electric effects in functionally stepped magnetic nanobilayers on ferroelectric substrates: Observation and theory on the influence of interlayer exchange coupling. Journal of Applied Physics, 2014, vol. 115, pp. 193909-193908. DOI: 10.1063/1.4878458.

7. More-Chevalier J., Lьders U., Cibert C., Nosov A., Domengus B., Bouregba R., Poullain G. Magnetoelectric coupling in $\mathrm{Pb}(\mathrm{Zr}, \mathrm{Ti}) \mathrm{O} 3-\mathrm{Galfenol}$ thin film heterostructures. Applied Physics Letters, 2015, vol. 107, pp. 252903-252906. DOI: 10.1063/1.4938218.

8. Sreenivasulu G., Fetisov L.Y., Fetisov Y.K., Srinivasan G. Piezoelectric single crystal langatate and ferromagnetic composites: Studies on low-frequency and resonance magnetoelectric effects. Applied Physics Letters, 2012, vol. 100, pp. 052901. DOI: 10.1063/1.3679661.

9. Sreenivasulu G., Qu P., Piskulich E., Petrov V.M., Fetisov Y.K., Nosov A.P., Qu H., Srinivasan G. Shear strain mediated magneto-electric effects in composites of piezoelectric lanthanum gallium silicate or tantalate and ferromagnetic alloys. Applied Physics Letters, 2014, vol. 105, pp. 32409-32408. DOI: 10.1063/1.4891536.

10. Available at: http://www.newpiezo.com/ru/langatate.html

11. Available at: http://www.gammamet.ru/ru/gm440a.htm

12. Atulasimha J., Flatau A.B. A review of magnetostrictive iron-gallium alloys. Smart Materials and Structures, 2011, vol. 20, pp. 043001. DOI: 10.1088/0964-1726/20/4/043001.

13. Jen S.U., Tsai T.L., Kuo P.C., Chi W.L., Cheng W.C. Magnetostrictive and structural properties of FeCoGa films. J. Appl. Phys., 2010, vol. 107, pp. 013914. DOI: 10.1063/1.3284962.

14. Available at: http://www.optosystems.ru/eng/index.php

15. Sreenivasulu G., Petrov V.M., Fetisov L.Y., Fetisov Y.K., Srinivasan G. Magnetoelectric interactions in layered composites of piezoelectric quartz and magnetostrictive alloys. Physical Review B, 2012, vol. 86, pp. 214405. DOI: 10.1103/PhysRevB.86.214405. 
Подана в журнал: 05.11.2018

УДК 537.633.9

DOI: $10.17804 / 2410-9908.2018 .6 .117-125$

\title{
ТОНКОПЛЕНОЧНЫЕ СТРУКТУРЫ FeGa-FeCoGa/Metglas/LGT ДЛЯ МАГНИТОЭЛЕКТРИЧЕСКИХ ДАТЧИКОВ МАГНИТНЫХ ПОЛЕЙ
}

\author{
А. П. Носов ${ }^{1,2,)^{*}}$, И. В. Грибов ${ }^{1, \text { б) }}$, Н. А. Москвина ${ }^{1, \text { в) }}$, \\ А. В. Дружинин ${ }^{1, \text { г) }}$, В. И. Осотов ${ }^{1, \text { д) }}$ \\ ${ }^{1}$ Институт физики металлов имени М.Н. Михеева Уральского отделения Российской академии наук, \\ ул. С. Ковалевской 18, Екатеринбург, 620108, Российская Федерация \\ ${ }^{2}$ Федеральное государственное автономное образовательное учреждение выстего образования «Уральский \\ федеральньй университет имени первого Президента России Б.Н. Ельцина», ул. Мира 19, Екатеринбург, \\ 62002, Российская Федераџия
}

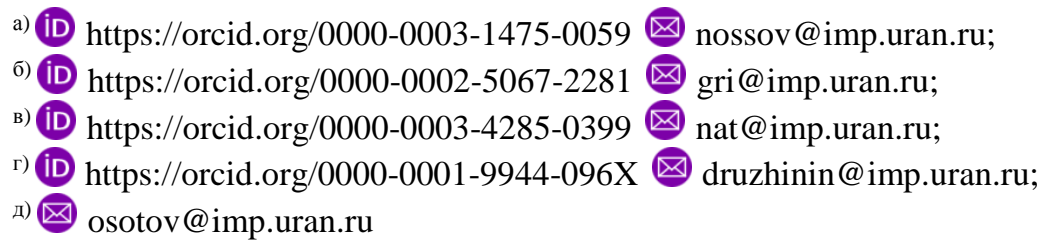

*Ответственный автор. Электронная почта: nossov@imp.uran.ru Адрес для переписки: ул. С. Ковалевской 18, Екатеринбург, 620108, Российская Федерация Тел.: +7 (343) 374-79-22; факс: +7 (343) 374-52-44

Исследовано влияние состава композитного магнитострикционного слоя на магнитоэлектрический эффект в трехслойных ламинатных структурах типа композитный магнитострикционный ферромагнетик (сегнетоэлектрик) композитный магнитострикционный ферромагнетик. В качестве сегнетоэлектрика использован монокристалл лантангаллиевого танталата $\mathrm{La}_{3} \mathrm{Ga}_{5,5} \mathrm{Ta}_{0,5} \mathrm{O}_{14}$. Композитный магнитострикционный ферромагнетик состоял из аморфной ленты сплава типа «Метглас», на поверхность которой импульсным лазерным осаждением были нанесены тонкие магнитострикционные пленки типа «галфенол» составов $\mathrm{Fe}_{0,72} \mathrm{Ga}_{0,28}$ или $\mathrm{Fe}_{0,62} \mathrm{Co}_{0,19} \mathrm{Ga}_{0,19}$. Исследованы зависимости магнитоэлектрического эффекта от статических и переменных магнитных полей в диапазоне частот от 20 Гц до 10 кГц и магнитных шумов в области частот 0,5-14 Гц. Экспериментально показано, что нанесение магнитострикционных пленок состава $\mathrm{Fe}_{0,62} \mathrm{Co}_{0,19} \mathrm{Ga}_{0,19}$ позволяет увеличить величину магнитоэлектрического коэффициента по напряжению и снизить уровень магнитных шумов. Результаты могут быть использованы для разработки магнитоэлектрических датчиков статических и переменных магнитных полей в системах магнитного неразрушающего контроля, эксплуатируемых при повышенных температурах.

Ключевые слова: неразрушающий контроль, датчик магнитного поля, магнитоэлектрический эффект, аморфный сплав, тонкие пленки, галфенол, лантангаллиевый танталат.

\section{1. Введение}

Разработка датчиков статических и переменных магнитных полей, работающих на новых принципах, является чрезвычайно важной для магнитного неразрушающего контроля. Такие датчики, особенно пригодные для работы в условиях повышенных температур, могут найти широкое применение для решения актуальных проблем неразрушающего контроля, прогнозировании ресурса и испытаний перспективных конструкционных и функциональных материалов. Достигнутые в последние годы успехи в исследованиях магнитоэлектрического эффекта (МЭ) в композитных планарных структурах с механически связанными слоями из 
магнитострикционного ферромагнитного и сегнетоэлектрического материалов [1] позволяют говорить о перспективности разработки датчиков этого типа для нужд магнитного неразрушающего контроля и диагностики. Если такую структуру поместить в статическое магнитное поле $H$, то в присутствии переменного магнитного поля $d H_{a c}$ будет наблюдаться прямой МЭ: при воздействии переменных магнитных полей за счет магнитострикции в ферромагнитном слое возникнет механическая деформация, которая приведет к генерации зарядов и возникновению электрического поля $d E$ в сегнетоэлектрическом слое вследствие его пьезоэлектрических свойств. Величина МЭ характеризуется магнитоэлектрическим коэффициентом по напряжению (МЭКН), который определяют как $\alpha=d E / H_{a c}=d V /\left(t d H_{a c}\right)$, где $d V-$ величина возникающего электрического напряжения; $t$ - толщина сегнетоэлектрического слоя. Значение $\alpha$ определяется физическими характеристиками как ферромагнитного слоя, в частности пьезомагнитным коэффициентом $q=d \lambda / d H$, где $\lambda$ - коэффициент магнитострикции; $H$ - статическое магнитное поле, так и сегнетоэлектрического слоя, в частности пьезомодулем $d$ и относительной диэлектрической проницаемостью $\varepsilon$. Для нужд неразрушающего контроля необходимы МЭ структуры с большой величиной МЭКН. Это может быть достигнуто оптимизацией характеристик магнитострикционной подсистемы за счет величины $q$ см. [2]. Повысить $q$ можно использованием многослойных магнитострикционных структур со слоями из материалов, имеющих разные знаки коэффициента магнитострикции, т. е. слоев с большим градиентом коэффициента магнитострикции по толщине. Для композитных объемных структур с ферромагнитными слоями эти результаты получили экспериментальное подтверждение в работе [3].

В качестве магнитострикционных слоев композитных МЭ структур очень часто используются магнитострикционные ленты аморфных сплавов класса «Метглас» (Metglas) [4]. Этот класс аморфных магнитных материалов характеризуется большим значением $q$, малым коэрцитивным полем, большой начальной магнитной проницаемостью, положительным значением $\lambda$. Улучшить магнитострикционные свойства таких аморфных лент можно путем нанесения на них тонких пленок магнитострикционных материалов, имеющих другой знак коэффициента магнитострикции, т. е. созданием композитных структур с градиентом $q$ по толщине. Ранее возможности изменения магнитных свойств аморфных лент сплавов типа «Метглас» были исследованы при нанесении на них тонких пленок немагнитных металлов [5].

В настоящее время в МЭ-структурах в качестве материалов сегнетоэлектрических слоев используются, как правило, различные типы пьезокерамик на основе свинца, такие как $\mathrm{PbMg}_{1 / 3} \mathrm{Nb}_{2 / 3} \mathrm{O}_{3}-\mathrm{PbTiO}_{3}$ (PMN-PT) [6] или $\mathrm{Pb}(\mathrm{Zr}, \mathrm{Ti}) \mathrm{O}_{3}$ (PZT) [7], основным достоинством которых является большая величина $d$. Однако для них характерны сравнительно узкий диапазон рабочих температур и низкая температура Кюри (не выше $350{ }^{\circ} \mathrm{C}$ ). В последние годы все более ужесточаются требования к охране окружающей среды и, как следствие, становится актуальным переход к пьезокерамикам, не содержащим свинец. Недавно в $[8,9]$ были представлены экспериментальные результаты по МЭ-структурам с сегнетоэлектрическим материалом состава $\mathrm{La}_{3} \mathrm{Ga}_{5,5} \mathrm{Ta}_{0,5} \mathrm{O}_{14}$ (лантангаллиевый танталат, LGT), не содержащим свинец. Достоинством LGT является возможность работы при высоких температурах, поскольку его температура плавления составляет $1450{ }^{\circ} \mathrm{C}$ и вплоть до этой температуры отсутствуют фазовые переходы. В качестве материала магнитострикционных слоев в работах $[8,9]$ использовали объемный сплав $\mathrm{Fe}-\mathrm{Co}-\mathrm{V}$ (пермендюр).

В настоящей работе проведен сравнительный анализ использования тонких пленок сплавов системы галфенол составов $\mathrm{Fe}_{0,72} \mathrm{Ga}_{0,28}$ и $\mathrm{Fe}_{0,62} \mathrm{Co}_{0,19} \mathrm{Ga}_{0,19}$ для создания композитных структур с градиентом коэффициента магнитострикции слоя по толщине и улучшения МЭКН в трехслойных ламинатных структурах типа композитный магнитострикционный ферромагнетик /сегнетоэлектрик/ композитный магнитострикционный ферромагнетик. В качестве сегнетоэлектрика использована монокристаллическая пластина лантангаллиевого танталата LGT. 


\section{2. Материал и методика}

В качестве сегнетоэлектрических слоев использовали пластины монокристалла лантангаллиевого танталата $\mathrm{La}_{3} \mathrm{Ga}_{5,5} \mathrm{Ta}_{0,5} \mathrm{O}_{14}$ (LGT, X-срез) с типичными размерами $5 \times 5 \times 0,3 \mathrm{~mm}^{3}$ [10]. В качестве магнитострикционного сплава класса «Метглас» использовали аморфные ленты состава 440A [11] (компания «Гаммамет», Екатеринбург, Россия) толщиной 34 мкм без термообработок. Статические измерения магнитострикции были проведены с использованием тензодатчика. Значения коэффициента магнитострикции насыщения измеряли при двух ориентациях магнитного поля $H$ : вдоль $\left(\lambda_{11}=20 \times 10^{-6} /\right.$ Э) и поперек $\left(\lambda_{12}=-15 \times 10^{-6} / Э\right)$ направления прокатки. Из этих данных была определена величина пьезомагнитного коэффициента $q=q_{11}+q_{12}=d \lambda_{11} / d H+d \lambda_{12} / d H$. Для лент состава $440 \mathrm{~A}$ было получено значение $q=0,46 \times 10^{-6} / Э$.

Магнитострикционные слои с композитной структурой получали нанесением на поверхность аморфной ленты состава 440A тонких пленок $\mathrm{Fe}_{0,72} \mathrm{Ga}_{0,28}$ или $\mathrm{Fe}_{0,62} \mathrm{Co}_{0,19} \mathrm{Ga}_{0,19}$ методом импульсного лазерного осаждения. Выбор этих составов обусловлен тем, что для объемных материалов системы $\mathrm{Fe}_{1-\mathrm{X}} \mathrm{Ga}_{\mathrm{x}}$ (галфенол) сплавы с $X=0,19$ или 0,28 имеют максимальную величину коэффициента магнитострикции [12]. При этом магнитострикция в сплавах $\mathrm{Fe}_{1-\mathrm{X}} \mathrm{Ga}$ х отрицательна. Легирование галфенола кобальтом позволяет увеличить значение $\lambda$. По данным работы [13], для тонких пленок сплавов $\mathrm{Fe}-\mathrm{Co}-\mathrm{Ga}$ состав $\mathrm{Fe}_{0,62} \mathrm{Co}_{0,19} \mathrm{Ga}_{0,19}$ имеет максимальное значение $\lambda$. Пленки толщиной 25 нм наносили с использованием эксимерного лазера CL 7050 (компания «Оптисистемы», г. Троицк, Московская область, Россия [14]).

Были исследованы трехслойные ламинатные структуры, в которых средним сегнетоэлектрическим слоем являлась пластина LGT. В качестве верхнего и нижнего слоев использовали либо аморфные магнитострикционные ленты 440А (далее - образцы 440А), либо аморфные магнитострикционные ленты 440A с пленкой $\mathrm{Fe}_{0,72} \mathrm{Ga}_{0,28}$ ((далее - образцы $440 \mathrm{~A} / \mathrm{FeGa})$, либо аморфные магнитострикционные ленты $440 \mathrm{~A}$ с нанесенной пленкой $\mathrm{Fe}_{0,62} \mathrm{Co}_{0,19} \mathrm{Ga}_{0,19}$ (далее - образцы $440 \mathrm{~A} / \mathrm{FeCoGa}$ ). Ламинатную структуру формировали склеиванием слоев клеем на основе цианокрилата. Ранее в [15] было показано, что при переходе от двухслойных структур к трехслойным величина МЭКН увеличивается.

МЭКН исследовали при приложении к ламинатной структуре статического $H$ и переменного $H_{a c}$ магнитных полей. Напряжение $d V$, возникающее в сегнетоэлектрическом слое, измеряли синхронным детектором на частоте $H_{a c}$. Величину МЭКН вычисляли по формуле $\alpha=d E / H_{a c}=d V /\left(t d H_{a c}\right)$, где $t-$ толщина сегнетоэлектрического слоя. Исследовали изменения $\alpha$ в зависимости от величины статического магнитного поля $H$ и частоты $f$ переменного магнитного поля.

Измерения магнитных шумов проводили в камере с трехслойным магнитным экраном при приложении к образцу переменного магнитного поля напряженностью 100-900 микроэрстед и усиления напряжения с образца предусилителем с большим импедансом и низким уровнем собственных шумов (типа SRS SR560). Затем сигнал подавали на анализатор спектра модели SRS SR780.

Все измерения были выполнены при комнатной температуре.

\section{3. Результаты и обсуждение}

На рис. 1 представлены результаты, полученные для структуры с аморфной лентой 440A на частотах 20, 110, 1000 Гц и 10 кГц. На всех частотах зависимости $\alpha(H)$ имеют схожую форму с максимумом при $H \approx 65$ Э, соответствующим максимуму полевой зависимости коэффициента $q$. Положение максимума практически не зависит от частоты. Максимальная величина $\alpha_{\max }$ при $H \approx 65$ Э сильно изменяется с частотой. Для фиксированной частоты с ростом $H$ значения $\sigma$ постепенно уменьшаются и становятся очень малыми при $H>200 Э$. Ход зависимости $\sigma(H)$ качественно соответствует виду зависимости $q(H)$. Для структур с плен- 
ками $\mathrm{Fe}_{0,72} \mathrm{Ga}_{0,28}$ или $\mathrm{Fe}_{0,62} \mathrm{Co}_{0,19} \mathrm{Ga}_{0,19}$ были получены аналогичные по форме зависимости $\alpha(H)$. Нанесение пленок не изменяло положение максимумов зависимостей $\alpha_{\max }(f)$ при $H \approx 65$ Э, однако влияло на величину максимального значения $\sigma$ во всем исследованном частотном диапазоне. Для решения задач магнитного неразрушающего контроля существенно, что величина МЭКН линейно зависит от $H$ в области полей 20-50 Э. Кроме того, зависимости $\alpha(H)$ нечетны по полю, что позволяет по знаку МКЭН судить о взаимной ориентации датчика и $H$.

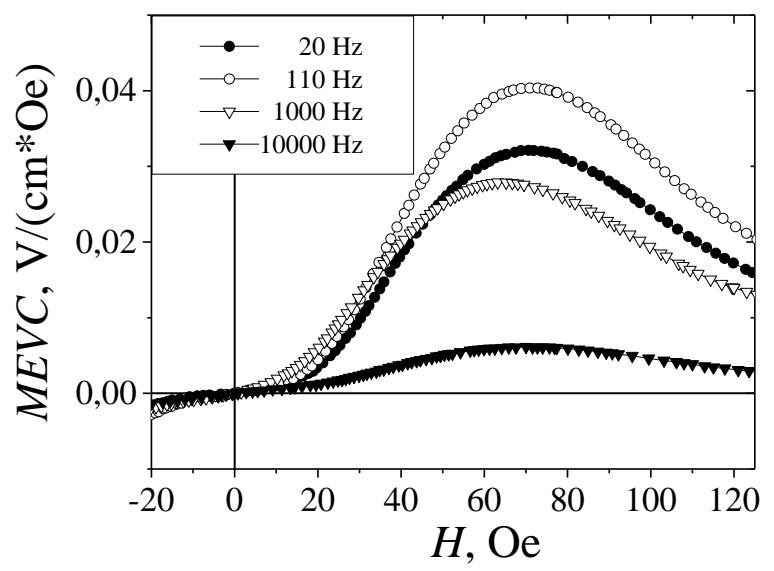

Рис. 1. Зависимости МЭКН от статического магнитного поля для значений частот переменного магнитного поля 20; 110; 1000 Гц и 10 кГц. Данные для структуры с аморфной лентой 440A

Частотные зависимости МЭКН в поле $H \approx 65$ Э для исследованных образцов представлены на рис. 2. Нанесение пленок по-разному влияет на величину $\alpha_{\max }(f)$ : для композитных структур с пленками состава $\mathrm{Fe}_{0,72} \mathrm{Ga}_{0,28}$ наблюдается уменьшение, а для структур с пленками состава $\mathrm{Fe}_{0,62} \mathrm{Co}_{0,19} \mathrm{Ga}_{0,19}$ - увеличение значений $\alpha_{\text {max }}(f)$ во всем исследованном интервале частот.

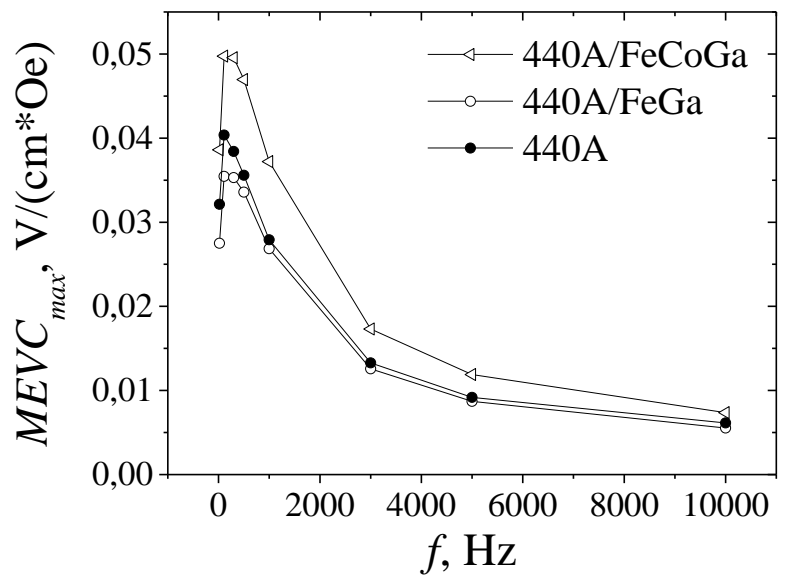

Рис. 2. Сравнение частотных зависимостей максимальной величины МЭКН для структур с магнитострикционными слоями только с аморфной лентой (440A), аморфными лентами с пленками $\mathrm{Fe}_{0,72} \mathrm{Ga}_{0,28}(440 \mathrm{~A} / \mathrm{FeGa})$ и $\mathrm{Fe}_{0,62} \mathrm{Co}_{0,19} \mathrm{Ga}_{0,19}(440 \mathrm{~A} / \mathrm{FeCoGa})$

Для возможных применений МЭ-ламинатных структур в качестве датчиков статических и переменных магнитных полей большое значение имеет линейность зависимостей $\alpha\left(H_{d c}\right)[13]$. В общем случае на зависимостях $\alpha\left(H_{d c}\right)$ можно выделить две области линейности с соответствующими коэффициентами: вблизи нулевого поля, которую можно характеризо- 
вать коэффициентом линейности $d M E V C / d H_{l o w H}$, и для больших (20-50 Э) полей - с коэффициентом линейности $d M E V C / d H_{h i g h H}$ (рис. 3). Коэффициент линейности вблизи нулевого поля представляет интерес для разработки МЭ датчиков неразрушающего контроля, предназначенных для регистрации предельно слабых статических и переменных полей. Коэффициент линейности в области больших (20-50 Э) полей может быть использован при разработке датчиков полей, работающих в условиях создания стационарного поля смещения, например за счет магнитной системы, обеспечивающей проведения измерений в постоянном по времени поле смещения в зоне контроля.

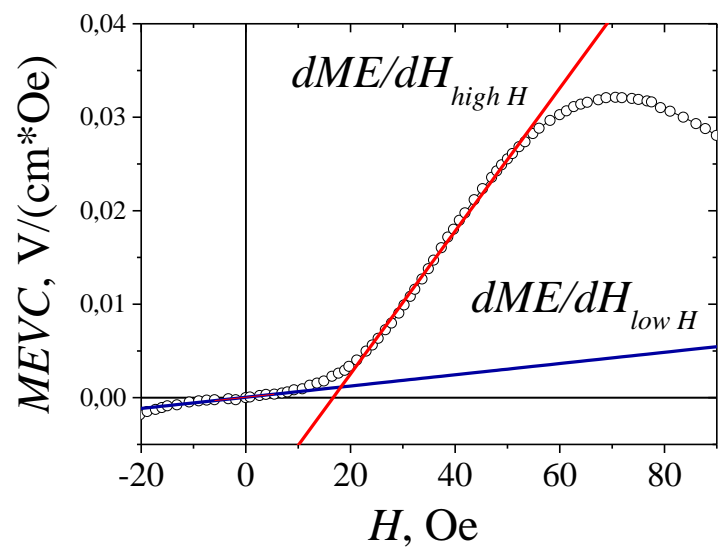

Рис. 3. Определения коэффициентов линейности МЭКН в области малых $\left(d M E V C / d H_{l o w H}\right)$ и больших $\left(d M E V C / d H_{h i g h H}\right)$ полей

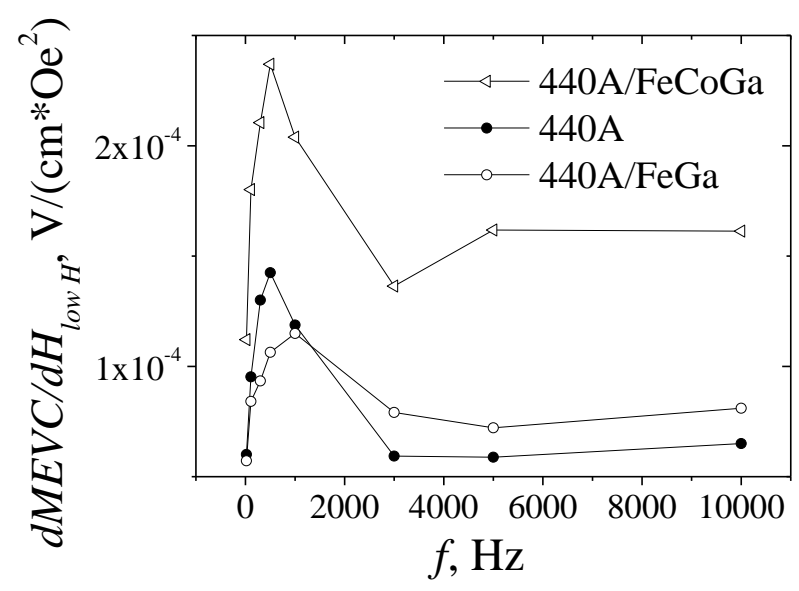

$a$

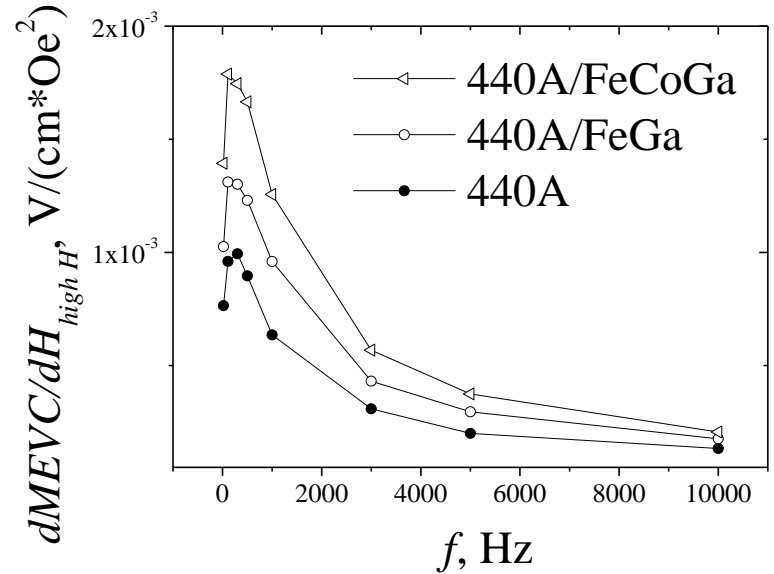

б

Рис. 4. Частотные зависимости коэффициентов линейности в области полей $(a)$ и больших (20-50 Э) полей (б) для структур с магнитострикционными слоями только с аморфной лентой (440A), аморфными лентами с пленками $\mathrm{Fe}_{0,72} \mathrm{Ga}_{0,28}(440 \mathrm{~A} / \mathrm{FeGa})$

$$
\text { и } \mathrm{Fe}_{0,62} \mathrm{Co}_{0,19} \mathrm{Ga}_{0,19}(440 \mathrm{~A} / \mathrm{FeCoGa})
$$

На рис. 4 представлены частотные зависимости коэффициентов линейности МЭламинатных структур. Оба коэффициента максимальны в случае нанесения тонких пленок $\mathrm{Fe}_{0,62} \mathrm{Co}_{0,19} \mathrm{Ga}_{0,19}$ во всем исследованном интервале частот. Нанесение пленок $\mathrm{Fe}_{0,72} \mathrm{Ga}_{0,28}$ приводит к росту коэффициента линейности в области больших полей, по сравнению с образцом только с аморфной лентой, однако для области вблизи нулевых полей наблюдается обратная зависимость. 
Качественно наблюдаемые закономерности можно интерпретировать с учетом того, что для ламинатных структур величина МЭКН пропорциональна отношению $d_{11} / \varepsilon_{11}$. В случаe LGT относительная диэлектрическая проницаемость $\varepsilon_{11}$ слабо зависит от частоты в диапазоне до 10 кГц. Поэтому изменения МЭКН с частотой качественно могут быть объяснены частотными изменениями характеристик ферромагнитных слоев. Коэффициент магнитомеханической связи определяется как $k_{m}=\left(4 \pi \lambda^{\prime} \mu_{r}^{\prime} / E\right)^{1 / 2}$, где $\lambda^{\prime}-$ коэффициент магнитострикции в переменном магнитном поле, который в свою очередь пропорционален $q ; \mu_{r}^{\prime}-$ действительная часть относительной магнитной проницаемости; $E$ - модуль Юнга. Наблюдаемое увеличение максимального значения $\alpha$ во всем исследованном диапазоне частот для структур с пленкой $\mathrm{Fe}_{0,62} \mathrm{Co}_{0,19} \mathrm{Ga}_{0,19}$, по-видимому, связано с увеличением $k_{m}$ за счет $q$ из-за разных знаков $\lambda$ в аморфном сплаве и пленке. Уменьшение максимального значения $\alpha$ для структур с пленкой $\mathrm{Fe}_{0,72} \mathrm{Ga}_{0,28}$ может быть связано с влиянием текстуры.

На рис. 5 приведены результаты измерений магнитных шумов в области частот 0,5-14 Гц. Видно, что градиент магнитострикции в магнитострикционных слоях существенно снижает уровень шумов структуры. При этом для частоты 1 Гц уровень шумов в структуре с магнитострикционными слоями аморфная лента/пленка $\mathrm{Fe}_{0,62} \mathrm{Co}_{0,19} \mathrm{Ga}_{0,19}$ почти в три раза ниже, а в структуре аморфная лента/пленка $\mathrm{Fe}_{0,72} \mathrm{Ga}_{0,28}$ почти в два раза ниже, чем в структуре только с аморфной лентой.

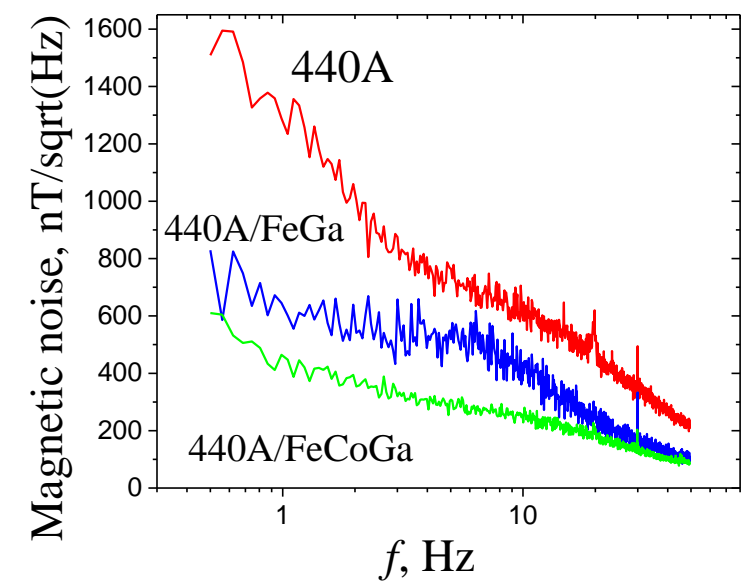

Рис. 5. Сравнение частотных зависимостей максимальной величины МЭКН для структур с магнитострикционными слоями только с аморфной лентой (440A), аморфными лентами с пленками $\mathrm{Fe}_{0,72} \mathrm{Ga}_{0,28}(440 \mathrm{~A} / \mathrm{FeGa})$ и $\mathrm{Fe}_{0,62} \mathrm{Co}_{0,19} \mathrm{Ga}_{0,19}(440 \mathrm{~A} / \mathrm{FeCoGa})$

Из совокупности полученных данных можно сделать вывод о том, что для решения задач неразрушающего контроля предпочтительно использование ламинатных МЭ-структур на основе композитных ферромагнитных магнитострикционных слоев типа аморфная лента сплава $440 \mathrm{~A} /$ тонкая пленка $\mathrm{Fe}_{0,62} \mathrm{Co}_{0,19} \mathrm{Ga}_{0,19}$.

\section{4. Заключение}

Исследован магнитоэлектрический эффект (МЭ) в ламинатных структурах типа композитный магнитострикционный ферромагнетик /сегнетоэлектрик/ композитный магнитострикционный ферромагнетик с монокристаллическими пластинами лантангаллиевого танталата LGT в качестве сегнетоэлектрика. Композитные ферромагнитные магнитострикционные слои с градиентом коэффициента магнитострикции по толщине были получены импульсным лазерным осаждением тонких пленок $\mathrm{Fe}_{0,72} \mathrm{Ga}_{0,28}$ и $\mathrm{Fe}_{0,62} \mathrm{Co}_{0,19} \mathrm{Ga}_{0,19}$ с отрицательным коэффициентом магнитострикции на аморфные ленты типа «Метглас» с положительным ко- 
эффициентом магнитострикции. Нанесение тонких пленок $\mathrm{Fe}_{0,62} \mathrm{Co}_{0,19} \mathrm{Ga}_{0,19}$ приводит к увеличению максимальной величины магнитоэлектрического коэффициента по напряжению и коэффициентов линейности во всем исследованном диапазоне частот, а также снижению уровня магнитных шумов. Ламинатные структуры на основе LGT с композитными магнитострикционными слоями из $\mathrm{Fe}_{0,62} \mathrm{Co}_{0,19} \mathrm{Ga}_{0,19}$ могут представлять интерес для решения задач магнитного неразрушающего контроля при повышенных температурах.

\section{Благодарность}

Работа выполнена в рамках государственного задания Минобрнауки России (тема «Сnuн», № AAAA-A18-118020290104-2).

\section{Литература}

1. Lawes G., Srinivasan G. Introduction to magnetoelectric coupling and multiferroic films // J. Phys. D: Appl. Phys. - 2011. - Vol. 44. - P. 243001. - DOI: 10.1088/0022-3727/44/24/243001.

2. Petrov V. M., Srinivasan G. Enhancement of magnetoelectric coupling in functionally graded ferroelectric and ferromagnetic bilayers // Phys. Rev. B. - 2008. - Vol. 78. - P. 184421. DOI: 10.1103/PhysRevB.78.184421.

3. Flexural deformation in a compositionally stepped ferrite and magnetoelectric effects in a composite with piezoelectrics / S. K. Mandal, G. Sreenivasulu, V. M. Petrov, G. Srinivasan // Appl. Phys. Lett. - 2010. - Vol. 96. - P. 192502. - DOI: 10.1063/1.3428774.

4. Magnetic Alloy 2605SA1 (iron-based) // Tech. Bulletin. ref: 2605SA106192009. - Metglas Inc., Conway, SC, 2009.

5. Out-of-plane anisotropy and low field induced magnetic domain reorientation in Al/Metglas-2605S2/Al trilayer sensors / E. C. Passamani, C. Larica, P. S. Moscon, P. M. Zelis, F. H. Sanchez // J. Appl. Phys. - 2011. - Vol. 110. - P. 043906. - DOI: 10.1063/1.3622339.

6. Magneto-electric effects in functionally stepped magnetic nanobilayers on ferroelectric substrates: Observation and theory on the influence of interlayer exchange coupling / I. V. Gribov, V. I. Osotov, A. P. Nosov, V. M. Petrov, G. Sreenivasulu, G. Srinivasan // Journal of Applied Physics. - 2014. - Vol. 115. - P. 193909-193908. - DOI: 10.1063/1.4878458.

7. Magnetoelectric coupling in $\mathrm{Pb}(\mathrm{Zr}, \mathrm{Ti}) \mathrm{O} 3-$ Galfenol thin film heterostructures / J. MoreChevalier, U. Lьders, C. Cibert, A. Nosov, B. Domengus, R. Bouregba, G. Poullain // Applied Physics Letters. - 2015. - Vol. 107. - P. 252903-252906. - DOI: 10.1063/1.4938218.

8. Piezoelectric single crystal langatate and ferromagnetic composites: Studies on lowfrequency and resonance magnetoelectric effects / G. Sreenivasulu, L. Y. Fetisov, Y. K. Fetisov, G. Srinivasan // Applied Physics Letters. - 2012. - Vol. 100. - P. 052901. - DOI: 10.1063/1.3679661.

9. Shear strain mediated magneto-electric effects in composites of piezoelectric lanthanum gallium silicate or tantalate and ferromagnetic alloys / G. Sreenivasulu, P. Qu, E. Piskulich., V. M. Petrov, Y. K. Fetisov, A. P. Nosov, H. Qu, G. Srinivasan // Applied Physics Letters. - 2014. Vol. 105. - P. 32409-32408. - DOI: 10.1063/1.4891536.

10. URL: http://www.newpiezo.com/ru/langatate.html

11. URL: http://www.gammamet.ru/ru/gm440a.htm

12. Atulasimha J., Flatau A. B. A review of magnetostrictive iron-gallium alloys // Smart Mater. Struct. - 2011. - Vol. 20. - P. 043001. - DOI: 10.1088/0964-1726/20/4/043001.

13. Magnetostrictive and structural properties of FeCoGa films / S. U. Jen, T. L. Tsai, P. C. Kuo, W. L. Chi, W. C. Cheng // J. Appl.Phys. - 2010. - Vol. 107. - P. 013914. DOI: $10.1063 / 1.3284962$.

14. URL: http://www.optosystems.ru/eng/index.php

15. Magnetoelectric interactions in layered composites of piezoelectric quartz and magnetostrictive alloys / G. Sreenivasulu, V. M. Petrov, L. Y. Fetisov, Y. K. Fetisov, G. Srinivasan // Physical Review B. 2012. - Vol. 86. - P. 214405. - DOI: 10.1103/PhysRevB.86.214405. 